\title{
Review of Research on critical velocity in tunnel fire
}

\author{
PEI Gui-hong ${ }^{1,2}$, ZHANG Qiu-yi ${ }^{1, *}$ \\ ${ }^{1}$ School of civil engineering and architecture, Southwest Petroleum University, 610500 Chengdu, China; \\ ${ }^{2}$ School of Architectural Economics and Engineering Management, Hubei Business College, 430073Wuhan, China
}

\begin{abstract}
The critical velocity is the key for tunnel fire control. If the longitudinal ventilation velocity is greater than the critical velocity when the fire occurs, the upstream of the fire source is smokeless, and the smoke will flow to the downstream of the fire source, which can effectively control the fire spread and provide valuable time for personnel to escape and fire fighting. The researches of domestic and foreign scholars are used to investigate the influencing factors of critical velocity. the results show that the main influencing factors of critical velocity are fire heat release rate, tunnel section geometry, obstacle and slope in tunnel, etc. In this paper, the influencing factors are summarized, and some problems that need to be studied in tunnel fire are put forward.
\end{abstract}

\section{Introduction}

With the rapid development of Chinese economic construction, various railway tunnels, highway traffic tunnels and urban subway tunnels are developing quickly. According to a lot of fire literature investigation, the smoke is the main cause of casualties in tunnel fire. On the basis of fire statistics in the United Kingdom and Japan, more than 50 percent of the deaths were caused by fires smoke, because the fire will produce lots of smoke in the tunnel, under the effect of thermal buoyancy, the smoke will rise into tunnel ceiling and spread along the top of the tunnel, a large amount of high temperature of smoke will have an impact on the structure of the tunnel. The movement of smoke reduces the visibility of people in the tunnel and the high temperature of the smoke will cause casualties.

\section{The key factors of smoke control}

In the event of a fire in the tunnel, when there is no longitudinal ventilation, the smoke symmetrically spreads along the tunnel ceiling, as shown in figure 1, at the same time, there is a symmetrical circulation air movement on both sides of the fire source. In the presence of longitudinal ventilation, the smoke flows asymmetrically on both sides of the fire source, if the longitudinal ventilation velocity is less than the critical velocity, the upstream smoke of the fire source is not overcome, the phenomenon of back-layering will occur as shown in figure 2, therefore, the longitudinal ventilation velocity should be greater than the critical velocity, which makes the upstream region of the fire source is smokeless, thus the fire spread can be effectively controlled, as shown in figure 3 .

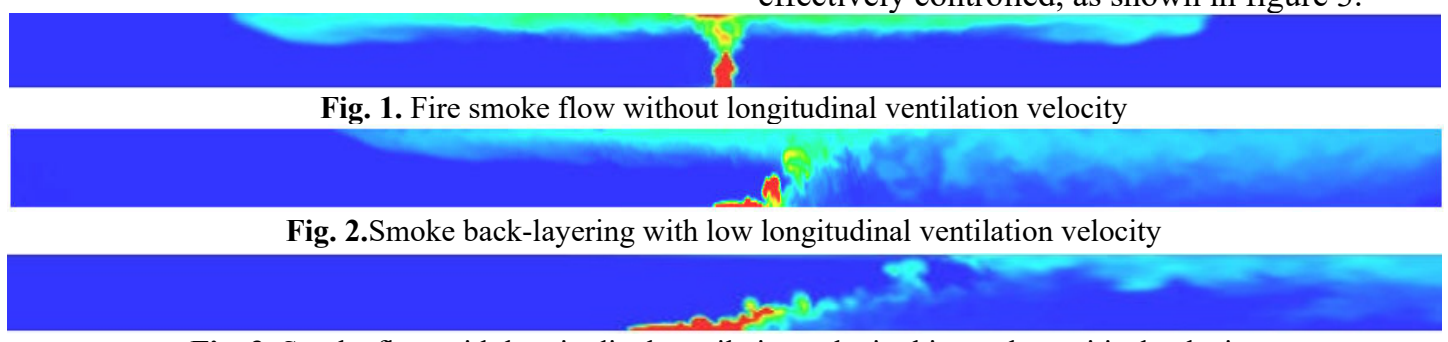

Fig. 3. Smoke flow with longitudinal ventilation velocity bigger than critical velocity

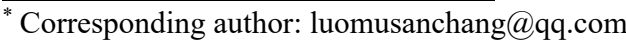


From the analysis of the above three diagrams, it can be seen that the key to controlling smoke of tunnel fire is critical velocity, there are many factors affecting critical velocity, such as fire heat release rate, tunnel section geometry, obstacles and slope, etc. In the last 40 years, scholars at home and abroad have done lots of research on the critical velocity, in this paper, the influence factors of critical velocity are summarized as follows.

\section{The influence of hear release rate on critical velocity}

In the 1860 s, the earliest tunnel fire tests were carried out in Switzerland, and the characteristics of the fires under different ventilation conditions were preliminarily studied, then nine western European countries carried out a series of large-scale tunnel fire tests in three tunnels in Germany, Finland and Norway according to different fire scales, the experimental results showed that the smoke movement was strongly influenced by longitudinal ventilation velocity.

On the basis of Froude number, the relationship between critical velocity and heat release rate was first obtained by Thomas[1,2], Thomas put forward critical Froude number to predict critical velocity, when the critical Froude number was equal to 1, the back-layering phenomenon disappeared, the prediction model of critical velocity was obtained:

$$
V_{c}=\left(\frac{g Q H}{\rho_{0} C_{p} T_{f} A}\right)^{1 / 3}
$$

Where $Q$ is the heat release rate, $\mathrm{W} ; V_{\mathrm{c}}$ is the critical velocity, $\mathrm{m} / \mathrm{s} ; \mathrm{g}$ is the acceleration of gravity, $\mathrm{m} / \mathrm{s}^{2} ; \mathrm{H}$ is the tunnel height, $\mathrm{m} ; \rho_{0}$ is the air density, $\mathrm{Kg} / \mathrm{m}^{3} ; C_{p}$ is the Specific heat at constant pressure, $\mathrm{J} / \mathrm{kg} \cdot \mathrm{K} ; T_{f}$ is the hot air temperature, $\mathrm{K}$; $\mathrm{A}$ is the cross-sectional area of tunnel, $\mathrm{m}^{2}$.

According to Froude number and test data, Heselden and Danziger, Kennedy[3] derived the critical velocity formula, which was also applied in the United States Department of Transportation Metro Environmental Simulation Project in 1982, the equation is as follows:

$$
\begin{gathered}
V_{c}=K_{g} k\left(\frac{g H Q}{\rho_{0} C_{p} A T_{f}}\right)^{1 / 3} \\
T_{f}=\frac{Q}{\rho_{0} C_{p} A V_{c}}+T_{0}
\end{gathered}
$$

Where $T_{0}$ is the ambient air temperature, $\mathrm{K} ; \mathrm{k}$ is the dimensionless coefficient, $\mathrm{k}=0.61 ; K_{\mathrm{g}}$ is the slope correction coefficient.

From the formulas (1) and (2), it can be seen that the critical velocity is directly proportional to the $1 / 3$ power of the heat release rate. However, experimental studies in recent years have shown that this kind of empirical formula has its limitations. In 1993, Bettis[4] conducted a full-scale test in a coal mine tunnel in Buxton, England. It was found that when the heat release rate is small, the critical velocity is directly proportional to the $1 / 3$ power of the fire heat release rate, and when the heat release rate is larger, Critical velocity is independent of fire heat release rate.

In 1996, the full-scale fire test results of Memorial tunnel in the United States also showed that when the fire heat release rate was $50-100 \mathrm{MW}$, the prediction of critical velocity by the above formula was higher than $5-15 \%$. Therefore, it can be concluded that the prediction of critical velocity by formulas (1) and (2) is not accurate when the fire heat release rate is large.

Oka and Athinson[5] made an experiment with a 1:10 scale model, the results of the above full-scale tests were also verified. Based on the Froude similarity criterion and the comparative analysis of experimental data, it was concluded that the critical velocity was proportional to the $1 / 3$ power of the heat release rate at the $Q^{*} \leqq 0.12$, and the critical velocity was independent of the heat release rate at the $\mathrm{Q}^{*}>0.12$, The dimensionless critical velocity prediction model is obtained:

$$
\begin{gathered}
V_{c}^{*}=\left\{\begin{array}{l}
K_{v}\left(\frac{Q^{*}}{0.12}\right)^{1 / 3} Q^{*} \leq 0.12 \\
K_{v} \quad Q^{*}>0.12
\end{array}\right. \\
Q^{*}=\frac{Q}{\rho_{0} C_{p} T_{a} \sqrt{g H^{5}}} \\
Q^{*}=\frac{Q}{\rho_{0} C_{p} T_{a} \sqrt{g H^{5}}}
\end{gathered}
$$

$\mathrm{T}_{\mathrm{a}}$ is the ambient air temperature, $\mathrm{K} ; \mathrm{K}_{\mathrm{v}}$ is a coefficient, $\mathrm{K}_{\mathrm{V}}=0.22-0.38$.

The above studies improve the theoretical calculation of critical velocity gradually, but these formulas are obtained with the height of tunnel as the characteristic length, but the influence of tunnel width on critical velocity is not considered.

\section{The influence of geometric shape on critical velocity}

Based on the Oka model[5], Wu and Bakar[6] studied the critical velocity of five tunnel sections with the same height and different width by means of experiments and numerical simulation, the hydraulic diameter was used to replace the tunnel height, the critical velocity prediction model is given:

$$
\begin{aligned}
& V_{c}^{\bullet}= \begin{cases}0.4\left(\frac{Q^{\bullet}}{0.2}\right)^{1 / 3} & Q^{*} \leq 0.2 \\
0.4 & Q^{*}>0.2\end{cases} \\
& Q^{\bullet}=\frac{Q}{\rho_{0} C_{p} T_{a} \sqrt{g \bar{H}^{5}}} \\
& V_{c}^{\bullet}=\frac{V_{c}}{\sqrt{g \bar{H}}}
\end{aligned}
$$

Where $\bar{H}$ is the hydraulic diameter, $\mathrm{m}$.

Li[7] carried out experiments in two tunnels with different cross-section sizes, the results showed that the critical velocity was only related to the dimensionless 
heat release rate, the dimensionless back-layering length was related to the dimensionless ventilation velocity and critical velocity, the dimensionless critical velocity prediction model is given:

$$
V_{c}^{\bullet}= \begin{cases}0.81 Q^{\bullet 1 / 3} & Q^{*} \leq 0.15 \\ 0.43 & Q^{*}>0.15\end{cases}
$$

Weng[8] studied the critical velocity and back-layering length with nine different tunnel section, compared the results with Wu model[6] and Li model[7], it was found that the values are bigger than latter two, critical velocity prediction model is shown in formula (11):

$$
V_{c}^{*}=0.82 Q^{* 1 / 3} \quad Q^{*} \leq 0.15
$$

\section{The influence of obstacles on critical velocity}

Later studies have tended to be more practical, when the fire occurs in the tunnel, critical velocity will be affected due to the obstacles, as shown in figure 4.

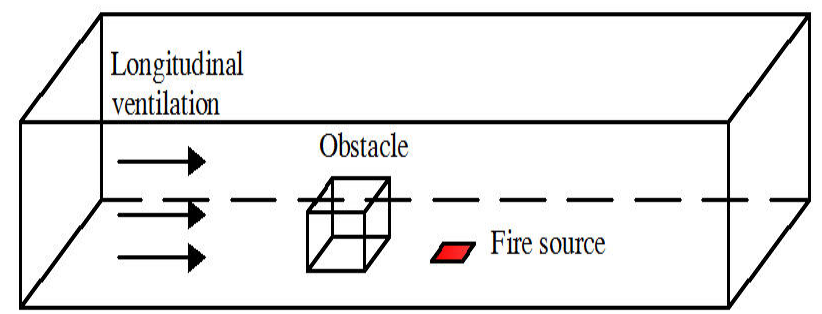

Fig. 4. Schematic diagram of longitudinal ventilation in tunnel with obstruction

Lee Y-P[9] researched the effect of vehicle congestion on the critical velocity by means of small-scale tests and numerical simulation, obstacle ratio range from $5 \%$ to $31 \%$ was set at the upstream of the fire source, the results showed that the velocity reduction rate was approximately equal to the obstacle ratio, the relation between the obstacle ratio and the ciritical velocity was presented as shown in formula (12):

$$
V_{c}^{\prime} / V_{c}=1-\phi
$$

Where the $V_{c}$ ' is the critical velocity with obstacle, $\mathrm{m} / \mathrm{s} ; V_{c}$ is the critical velocity without obstacle, $\mathrm{m} / \mathrm{s} ; \varnothing$ is the obstacle ratio, $\%$.

Kang[10] uses tunnel hydraulic diameter to replace tunnel height, on the basis of formula (2), the prediction formula of critical velocity was obtained:

$$
V_{C}=K_{g}\left(\frac{g \bar{H} Q}{F_{c} \rho_{0} C_{p} A T_{f}}\right)^{1 / 3}
$$

Li[11] brought $A_{\text {local }}=\mathrm{A}(1-\emptyset)$ to formula(2) and (13), and found that the trend of the result of CFD simulation was the same as the calculated value by formula (13), but the trend of formula (2) was opposite to CFD numerical simulation. However, the predicted result of by formula (13) was smaller than CFD simulation, by setting the fire source on the obstacle surface and comparing the experimental results of $\mathrm{Li}[7]$, a new prediction model was obtained by fitting, as shown in formula (14):

$$
V_{C}^{*}= \begin{cases}0.43\left(Q^{*} / 0.15\right)^{1 / 3} & , Q^{*} \leq 0.15 \\ 0.43 & , Q^{*}>0.15\end{cases}
$$

The positive and negative error of the prediction of this critical velocity was \pm 15 .

Gannouni[12] et al. numerically simulated the influence of the relative distance between the blockage and the tunnel ground on the critical velocity, it was concluded that when the relative distance increased, the critical velocity increased slightly, and when there was a blockage in the tunnel, the smoke back-layering length would be significantly reduced in the tunnel compared with that in the tunnel without blockage, the relationship between the longitudinal ventilation velocity and heat release rate was obtained:

$$
\begin{aligned}
V^{*} & =70.96 \exp \left(0.002 l^{*} / Q^{* 1 / 3}\right)+71.966 \\
L^{*} & =\frac{L}{H}
\end{aligned}
$$

Where $L^{*}$ is dimensionless back-layering length; $L$ is the back-layering length, m;

\section{The influence of slope on critical velocity}

In practice, many tunnels have slope ,as shown in figure 5 , many studies have showed that the smoke diffusion velocity in slope tunnel is different with horizontal tunnel, so the influence on critical velocity is different.

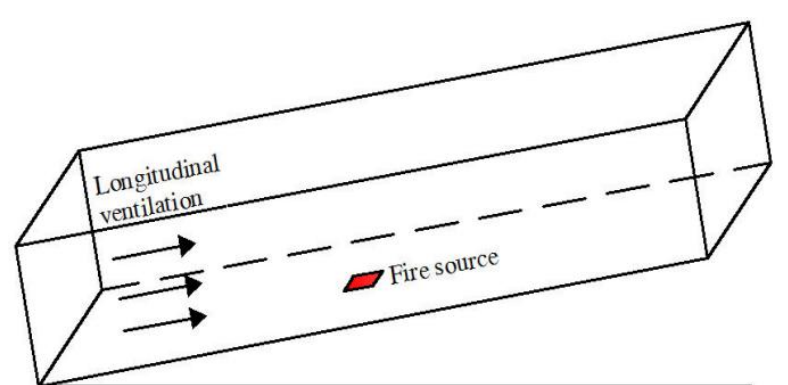

Fig.5.Schematic diagram of longitudinal ventilation in slope tunnel

Athinson and $\mathrm{Wu}[13]$ adopted an experimental method similar to that of Oka and Athinson[5], based on the critical velocity of Oka model, the influence of slope between $0^{\circ}$ and $10^{\circ}$ on critical velocity was analyzed, the expression was obtained:

$$
V_{c, \theta}=V_{c, 0} \cdot[1+0.014 \theta]
$$

$V_{c, \theta}$ is the critical velocity with slope, $\mathrm{m} / \mathrm{s} ; V_{c, 0}$ is the critical velocity without slope, $\mathrm{m} / \mathrm{s} ; \theta$ is the slope value.

Ko[14] developed an experiment with a 1:20 scale model, the experimental results showed that the critical velocity increased with the increasing of the slope at $0^{\circ}$, $2^{\circ}, 4^{\circ}, 6^{\circ}$ and $8^{\circ}$, and its expression is as follows:

$$
V_{c, \theta} / V_{c, 0}=(1+0.033 \theta)
$$

Yi[15] studied the influence of slope from $-3 \%$ to $3 \%$ on critical velocity by using the 1:10 scale model, 
compared with Wu model[6], good fit was obtained, the expression was obtained:

$$
V_{c, S} / V_{c, 0}=(1+0.034 S)
$$

$V_{c, S}$ is the critical velocity with slope, $\mathrm{m} / \mathrm{s} ; V_{c, 0}$ is the critical velocity without slope, $\mathrm{m} / \mathrm{s} ; S$ is tangent value of slope, $\%$.

Chow[16] studied the smoke movement characteristics at the slope of $0^{\circ}, 3^{\circ}, 6^{\circ}$ and $9^{\circ}$, by means of numerical simulation and small scale experiments, it was concluded that the critical velocity to prevent the smoke back-layering was higher in the slope tunnel, the expression is as follows:

$$
V_{c, \theta} / V_{c, 0}=(1+0.022 \theta)
$$

From the existing literature, most of these studies make fire source located in the center of the tunnel, and it is insufficient fro the accidental exploration of the location of the fire source, and the consideration and setting of the conditions are relatively simple, most of researches only consider the influence of one factor on the critical velocity, in general, the tunnel may be in a crowded state during a fire situation, and the fire may spread, when the radiation heat flux exceeds the critical radiant heat flux of the vehicle, the vehicle adjacent to the fire source will be easily ignited, at the same time , the single fire source will become multi-fire sources condition.

\section{7 conclusion and prospect}

A great deal of researches have been done on the characteristics and control of smoke movement in tunnel fire at home and abroad, and many prediction models of critical velocity have been established. However, there are still a large number of problems needed to be studied to provide guidance for fire prevention and evacuation of people in tunnel fires, this paper holds that the research on the critical velocity should be focus on the following aspects:

(1) In addition to the common factors, it should also be considered according to the actual situation, such as obstacle, slope, fire source location and so on, the current research basically consider a single factor and the fire source is located in the center of the tunnel, there are few researches about critical velocity under the condition of coexistence of multiple factors.

(2) Considering the actual situation, because the tunnel may be in the traffic crowded state during the fire, the fire exists the spreading phenomenon, when the radiation heat flux exceeds the critical radiation flux of the vehicle, the adjacent vehicle of the fire source is easy to be ignited, at this time, the single source of fire may become multi-fire sources condition, the heat release rate is definitely different from single fire source, and it must have a great influence on the critical velocity, the influence of the distance between multi-fire sources, blockage, slope and other factors on the critical velocity in multi-fire sources should be further studied.

(3) The larger the longitudinal ventilation velocity is, the smaller the smoke back-layering length is, but the fire may be stronger because of the bigger ventilation velocity

In order to ensure the safety of the tunnel, if the longitudinal ventilation velocity controls the smoke in the downstream of the fire source, but the heat release rate increases quickly to cause the danger of tunnel collapse, so we can reduce the critical velocity to reduce the risk, the influence of blockage, slope and tunnel geometry on the limited velocity is worth further studying.

\section{References}

1. Thomas P H. Fire Saf. J.(1958).

2. Thomas P H. Fire Res. Tech. Pap. 7,1( 1963)

3. Danziger N H, Kennedy W D. In: Proceedings of the 4th International Symposium Aerodynamics and Ventilation of Vehicle Tunnels. (York, UK 1982)

4. Bettis RJ, Jagger SF, Wu Y. The Health and Safety Executive (UK 1993)

5. Oka Y, Atkinson G T. Fire Saf. J., 25,305(1995)

6. Wu Y, Bakar M Z A. Fire Saf. J., 35,363(2000)

7. Li Y Z, Lei B, Ingason H. Fire Saf J, 45,361(2010)

8. Weng M C, Lu X L, Liu F, et al. Tunn. Undergr. Sp. Tech. 47,64 (2015)

9. Lee Y P, Tsai K C. Fire Saf. J.53,35(2012)

10. Lee Y P, Tsai K C. Fire Saf. J.53,35(2012)

11. Li L, Cheng X, Cui Y, et al. J. Fire Sci.30,413(2012)

12. Gannouni S, Maad R B. Tunn. Undergr. Sp. Tech. inc. Tren. Tech. Res. 48,147(2015)

13. Atkinson G T, Wu Y. Fire Saf. J. 27,335(1996)

14. Ko $\mathrm{G} \mathrm{H}$, Kim $\mathrm{S} \mathrm{R}$, Hong $\mathrm{S} \mathrm{R}$. J. Fire Sci. 28,27(2010)

15. Yi L, Xu Q, Xu Z, et al. Tunn. Undergr. Sp. Tech. 43,198(2014)

16. Chow W K, Gao Y, Zhao J H, et al. Fire Saf. J. 75,14(2015) 